\title{
LIBRI RICEVUTE E RECENSIONI
}

A. Foch - Mécanique Physique et Expérimentale. Tome $\mathrm{I}^{\mathrm{e}}$ : Statique et Résistarce des moariaux. Masson, Paris, 1961 ; pp. 326, fig. 210 , NF 55.

Partendo dalle due forme classiche del principio fondamentale della Statica l'A. mostra anzitutto, attraverso vari esempi, come esse possano essere utilizzate sia nei casi di equilibrio più semplici, sia nei sistemi articolati isostatici. Vari metodi di risoluzione, sia grafici che analitici, vi sono applicati.

Segue quindi una trattazione abbastanza ampia dei fenomeni di attrito, e delle conseguenze di essi in taluni problemi tecnici.

La seconda metà del volume è infine dedicata alla resistenza dei mate- riali e alla elasticità, con particolare riferimento alle applicazioni a taluni sistemi meccanici.

Un secondo volume, col sottotitolo "Dinamica fisica e sperimentale" è in preparazione.

Nel complesso si tratta di un opera di Meccanica applicata, cioè sviluppata a partire da principi che la meccanica razionale ha già discusso dimostrandone la validità, per cui scopo fondamentale dell'opera di Foch resta quello di mostrare come coi principi basilari della meccanica e con appropriati metodi di calcolo si possa prevedere il comportamento di taluni sistemi meccanici, quali si incontrano nella pratica. L'opera interessa pertanto più gli ingegneri ed $i$ tecnici, che i Fisici.

A. Drigo 